Cancer

\title{
Gene transfer: Bax to the future for cancer therapy
}

\section{N R Lemoine, I A McNeish}

\section{The Bax gene as a competitor in the marathon towards licensed cancer gene therapy}

lising

traperitoneal spread of gastrointestinal malignancies is a significant clinical problem and contributes to an incidence of distant relapse as high as $30 \%$ in gastric cancer. Local dissemination of tumour cells into the peritoneal cavity determines the outcome in advanced gastric cancer and diffusetype carcinoma, and patients with negative peritoneal washings have a more favourable prognosis. Extensive lymph node dissection has been shown (by quantitative reverse transcriptionpolymerase chain reaction for carcinoembryonic antigen and cytokeratin 20 combined with extensive intraoperative peritoneal lavage ${ }^{1}$ ) to open lymphatic channels and spread viable cancer cells into the peritoneal cavity. Hence patient subpopulations that might benefit from intraperitoneal therapy regimens may be identified.

Malignant disease localised within the abdominal cavity has been a target for the staged development of clinical gene therapy approaches because of the smaller doses of experimental agent and increased safety margins over systemic administration. There has been extensive-and safe-experience of $p 53$ gene therapy which culminated in a randomised phase III trial in which women with $p 53$ null or $p 53$ mutant ovarian cancer were randomised to chemotherapy alone or chemotherapy plus intraperitoneal Ad $p 53$ following optimum debulking primary surgery. However, the first interim analysis indicated that not only did Ad $p 53$ fail to improve effectiveness but it was also associated with increased toxicity. As a result, the study has been abandoned (reported in Zeimet and Marth $^{2}$ ). Two broad possibilities exist to explain why the trials were relatively unsuccessful. Firstly, there is the perennial problem of suboptimal gene transfer. Secondly, there is the possibility that $p 53$ is the "wrong" transgene. Although $p 53$ mutations are found in many malignancies ${ }^{3}$ and defective $p 53$ function may be causally linked to chemotherapy resistance, ${ }^{4}$ many aspects of $p 53$ biology remain obscure, especially factors involved in the decision that determines whether cells undergo apoptosis or cell cycle arrest in response to p53 activation. ${ }^{5}$ There is some evidence that low level $p 53$ expression, in the range likely after adenoviral gene transfer, causes cell cycle arrest rather than cell death. ${ }^{6}$ Also, the proapoptotic function of $p 53$ depends on transactivation of genes such as Bax, Apaf-1, Fas, and PTEN whose own expression or activity may be abnormal in tumour cells. ${ }^{7}$ Mutant $p 53$ can act as a dominant negative in $p 53$ tetramers, ${ }^{8}$ which could abrogate the effect of exogenous wild-type protein encoded by the transgene. Finally, polymorphisms of the $p 53$ gene (especially codon $72^{\text {arg }}$ ) can determine the responsiveness of tumours to chemo- and radiotherapy by influencing inhibition of p73. ${ }^{9}$ Hence while $p 53$ gene replacement was the early leader, it is likely to be overtaken by more robust competitors in the marathon towards a successful and licensed cancer gene therapy.

The work reported by Tsunemitsu and colleagues $^{10}$ in this issue of Gut focuses on the potential of the Bax gene as a strong inducer of apoptosis, targeting gastric cancer growing as solid tissue deposits and as intraperitoneal disease [see page 554]. They show that a replication defective adenoviral vector expressing human Bax as a transgene could induce death even of $p 53$ resistant gastric cancer. (Interestingly, treatment with a "control" virus expressing the reporter gene lac- $\mathrm{Z}$ was also observed to extend survival, a phenomenon that has been previously reported in the treatment of pancreatic cancer $\left.{ }^{11}\right)$. However, it was evident that penetration of tumour deposits was only superficial after intraperitoneal instillation and, even though survival of treated animals was extended, cure was not achieved. Thus it appears that while Bax may be a more effective transgene than $p 53$ for cancer gene therapy, its application might be restricted to lavage at the time of surgical dissection rather than the treatment of established bulk disease.

What further developments are on the horizon for the gene therapy of gastrointestinal cancers? We believe that advances will come from both improved gene delivery technologies and more powerful transgene combinations.

Replicating biological agents are the most promising means to improve the delivery kinetics in solid tumours, and both viruses and bacteria are being exploited for this purpose. The adenovirus $\mathrm{E} 1 \mathrm{~B} 55 \mathrm{kDa}$ protein suppresses p53 function in infected cells ${ }^{12}$ and E1B $55 \mathrm{~K}$ deleted adenoviral vectors replicate within and cause cytolysis of tumours with defective $\mathrm{p} 53$ function. ${ }^{13}$ In the past two years, six separate phase I/II trials of such a virus (variously known as dl1520, Onyx-015, and CI-1042) have been published, in a range of tumour types, including colorectal, ${ }^{14}{ }^{15}$ ovarian, ${ }^{16}$ and pancreatic carcinomas, ${ }^{17}{ }^{18}$ and in patients with liver metastases from gastrointestinal malignancies. ${ }^{19}$ In combination with chemotherapy, some responses were seen; with 5-fluorouracil, eight patients with colorectal liver metastases demonstrated either partial or minor responses, at least five of whom had previously been refractory to 5 -fluorouracil. ${ }^{13}{ }^{19}$ In primary pancreatic carcinoma, two patients had partial responses in combination with gemcitabine. ${ }^{17} \mathrm{~A}$ second generation of selectively replicating adenoviral vectors specifically targeting retinoblastoma $(\mathrm{Rb})$ function are approaching clinical trial. DNA tumour viruses such as adenovirus can infect and replicate in quiescent cells because viral proteins induce $S$ phase entry. The Gl-S phase cell cycle checkpoint is regulated by $\mathrm{pRb}$ and its related family members (p107, pl30), and efficient progression from G1 to $S$ phase requires binding and inactivation of the pRb family of proteins by the adenovirus ElA early viral protein. This interaction requires amino acids 121-127 of the E1A protein conserved region 2. Components of the Gl checkpoint, including $\mathrm{Rb}$ protein, cyclin $\mathrm{Dl}$, and pl6INK4a, are commonly altered in human malignancies, abrogating cell cycle control. Two similar adenovirus mutants have been described recently: dl922/947 is deleted in amino acids 122$129^{20}$ while $\Delta 24$ is deleted in amino acids 121-128. ${ }^{21}$ Both have been assessed in in vitro and in vivo models of cancer, and dl922/947 is capable of replicating with much greater efficiency within a panel of tumour cell lines (including gastrointestinal) than dl1520, with minimal S phase induction in quiescent non-immortalised cells. ${ }^{20}$ Transcriptional targeting of viral replication is also possible, and overexpression of cyclooxygenase 2 (COX-2) in gastrointestinal and pancreatic cancers has stimulated the construction and preclinical validation of selectively replicating adenoviruses incorporating the COX-2 promoter to drive expression of 
the viral early gene complex..$^{22}$ Improved delivery kinetics for adenoviral agents can be achieved by incorporating icodextrin solution in the carrier vehicle to prolong retention and distribution in the peritoneal cavity. ${ }^{23}$ More selective delivery of the viral agent can be achieved with bispecific single chain antibodies targeted toward epithelial cell adhesion molecule that is expressed on gastric tumours but not on normal gastric epithelium, allowing at least a 10-fold relative protection of normal tissues. ${ }^{24}$ Fibroblast growth factor receptors might also be exploited for targeting gastrointestinal tumours as it is perfectly feasible to incorporate receptor binding peptides into the coat of adenoviral particles. ${ }^{25}{ }^{26}$ Although less highly developed in clinical applications, oncolytic herpes viruses also look potentially useful for treating peritoneal malignancies. ${ }^{27}$ An intriguing possibility is that bacteria engineered to invade epithelial cells could be used to deliver therapeutic genes and proteins, and this might have particular application to gastrointestinal tumours. ${ }^{28}$

A variety of other transgenes with improved therapeutic potential are in development, including in combination with either chemotherapy or prodrug activating systems. Fhit expression is reduced in most cancers, and Fhit replacement by FHIT expression viruses in oesophageal and pancreatic cancers induces apoptosis in cancer cells. ${ }^{29}$ FHIT viral gene delivery prevents or retards development of carcinogen induced forestomach tumours and reverses development of established tumours in mice by $60-70 \%$ through an apoptotic pathway. Among the proapoptotic members of the family to which Bax belongs, its relative Bim is possibly the most powerful inducer of cell death, ${ }^{30} 31$ and combination gene therapy in which Bim was delivered in an adenoviral vector together with the herpes simplex virus thymidine kinase gene markedly enhanced its effectiveness. ${ }^{32}$ This complementary approach-combining viral and cellular elements-is further exemplified by a fusion protein containing a short peptide from the proapoptotic family member Bak fused to the herpes simplex protein VP22, which was shown to be very effective in entering cells and triggering apoptosis. ${ }^{33}$ It has also been shown that delivery of genes responsible for the downstream effector machinery of programmed cell deathSmac/DIABLO or caspase genes, for instance-can enhance sensitivity to chemotherapeutic agents that are otherwise ineffective. ${ }^{34} 35$

The work reported by Tsunemitsu and colleagues $^{10}$ in this issue supports the idea that apoptotic triggers will be useful in building cancer gene therapies of the future but it is clear that much effort is required for improved agent delivery and combinations with other therapeutic modalities to optimise their therapeutic effect.

Gut 2004;53:478-479.

doi: $10.1136 /$ gut. 2003.030312

\section{Authors' affiliations}

N R Lemoine, I A McNeish, Cancer Research UK Clinical Centre, Sir John Vane Science Building, Barts and the London School of Medicine and Dentistry, London, UK

Correspondence to: Professor N R Lemoine Cancer Research UK Clinical Centre, Sir John Vane Science Building, Barts and the London School of Medicine and Dentistry, Charterhouse Sq, London ECIM 6BQ, UK;

nick.lemoine@cancer.org.uk

\section{REFERENCES}

1 Marutsuka T, Shimada S, Shiomori K, et al. Mechanisms of peritoneal metastasis after operation for non-serosa-invasive gastric carcinoma: an ultrarapid detection system for intraperitoneal free cancer cells and a prophylactic strategy for peritoneal metastasis. Clin Cancer Res 2003;9:678-85

2 Zeimet AG, Marth C. Why did p53 gene therapy fail in ovarian cancer? Lancet Oncol 2003:4:415-22

3 Levine AJ. p53, the cellular gatekeeper for growth and division. Cell 1997;88:323-31.

4 Reles A, Wen WH, Schmider A, et al. Correlation of p53 mutations with resistance to platinumbased chemotherapy and shortened survival in ovarian cancer. Clin Cancer Res 2001; 7:2984-97

5 Vousden KH, Lu X. Live or let die: the cell's response to p53. Nat Rev Cancer 2002;2:594-604.

6 Chen X, Ko L, Jayaraman L, et al. p53 levels, functional domains, and DNA damage determine the extent of the apoptotic response of tumor cells Genes Dev 1996;10:2438-51.

7 Wolf BB, Schuler M, Li W, et al. Defective cytochrome c-dependent caspase activation in ovarian cancer cell lines due to diminished or absent apoptotic protease activating factor-1 activity. J Biol Chem 2001;276:34244-51.

8 de Vries A, Flores ER, Miranda B, et al. Targeted point mutations of $\mathrm{p} 53$ lead to dominant-negative inhibition of wild-type p53 function. Proc Natl Acad Sci U S A 2002;99:2948-53.

9 Bergamaschi D, Gasco M, Hiller L, et al. p53 polymorphism influences response in cancer chemotherapy via modulation of p73-dependent apoptosis. Cancer Cell 2003;3:387-402.

10 Tsunemitsu $Y$, Kagawa S, Tokunaga N, et al. Molecular therapy for peritoneal dissemination of xenotransplanted human MKN-45 gastric cancer cells with adenovirus mediated Bax gene transfer. Gut 2004;53:554-60.

11 Rigg AS, Lemoine NR. Adenoviral delivery of TIMP1 or TIMP2 can modify the invasive behavior of pancreatic cancer and can have a significant antitumor effect in vivo. Cancer Gene Ther 2001;8:869-78.

12 Barker DD, Berk AJ. Adenovirus proteins from both $E 1 B$ reading frames are required for transformation of rodent cells by viral infection and DNA transfection. Virology 1987; 156:107-21.

13 Heise C, Sampson-Johannes A, Williams A, et al. ONYX-015, an E1B gene attenuated adenovirus, causes tumor-specific cytolysis and antitumoral efficacy that can be augmented by standard chemotherapeutic agents. Nat Med 1997;3:639-44

14 Reid T, Galanis E, Abbruzzese J, et al. Intraarterial administration of a replication-selective adenovirus (dl1520) in patients with colorectal carcinoma metastatic to the liver: a phase I trial. Gene Ther 2001;8:1618-26.

15 Hamid O, Varterasian ML, Wadler S, et al. Phase II trial of intravenous $\mathrm{Cl}-1042$ in patients with metastatic colorectal cancer. J Clin Oncol 2003;21: 1498-504.

16 Vasey PA, Shulman LN, Campos S, et al. Phase I trial of intraperitoneal injection of the E1B-55-kdgene-deleted adenovirus ONYX-015 (dl1520) given on days 1 through 5 every 3 weeks in patients with recurrent/refractory epithelial ovarian cancer. J Clin Oncol 2002;20:1562-9.

17 Hecht JR, Bedford R, Abbruzzese JL, et al. A phase I/II trial of intratumoral endoscopic ultra sound injection of ONYX-015 with intravenous gemcitabine in unresectable pancreatic carcinoma. Clin Cancer Res 2003:9:555-61.

18 Mulvihill S, Warren R, Venook A, et al. Safety and feasibility of injection with an E1B-55 kDa gene-deleted, replication-selective adenovirus (ONYX-015) into primary carcinomas of the pancreas: a phase I trial. Gene Ther $2001 ; 8: 308-15$

19 Reid T, Galanis E, Abbruzzese J, et al. Hepatic arterial infusion of a replication-selective oncolytic adenovirus (d 1520): Phase II viral, immunologic, and clinical endpoints. Cancer Res 2002;62:6070-9.

20 Heise C, Hermiston T, Johnson L, et al. An adenovirus E1A mutant that demonstrates potent and selective systemic anti-tumoral efficacy. Nat Med 2000:6:1134-9.

21 Fueyo J, Gomez-Manzano C, Alemany R, et al. A mutant oncolytic adenovirus targeting the $\mathrm{Rb}$ pathway produces anti-glioma effect in vivo. Oncogene 2000:19:2-12.

22 Yamamoto $M$, Davydova J, Wang $M$, et al. Infectivity enhanced, cyclooxygenase- 2 promoterbased conditionally replicative adenovirus for pancreatic cancer. Gastroenterology 2003:125:1203-18.

23 Engler H, Machemer TR, Schluep T, et al. Development of a formulation that enhances gene expression and efficacy following intraperitoneal administration in rabbits and mice. Mol Ther 2003;7:558-64.

24 Heideman DA, van Beusechem VW Offerhaus GJ, et al. Selective gene transfer into primary human gastric tumors using epithelial cell adhesion molecule-targeted adenoviral vectors with ablated native tropism. Hum Gene Ther 2002; 13:1677-85.

25 Maruta F, Parker AL, Fisher KD, et al. Identification of FGF receptor-binding peptides for cancer gene therapy. Cancer Gene Ther 2002; $9: 543-52$

26 Lanciotti J, Song A, Doukas J, et al. Targeting adenoviral vectors using heterofunctional polyethylene glycol FGF2 conjugates. Mol Ther 2003;8:99-107.

27 Bennett JJ, Delman KA, Burt BM, et al Comparison of safety, delivery, and efficacy of two oncolytic herpes viruses (G207 and NV1020) for peritoneal cancer. Cancer Gene Ther 2002;9:935-45

28 Critchley RJ, Jezzard S, Radford KJ, et al. Potential therapeutic applications of recombinant invasive E. coli. Gene Ther 2004 (in press).

29 Ishii $\mathbf{H}$, Zanesi N, Vecchione A, et al. Regression of upper gastric cancer in mice by FHIT gene delivery. FASEB J 2003;17:1768-70.

30 Marani M, Hancock D, Lopes R, et al. Role of Bim in the survival pathway induced by Raf in epithelial cells. Oncogene 2004 (in press)

31 Marani M, Tenev T, Hancock D, et al. Identification of novel isoforms of the $\mathrm{BH} 3$ domain protein Bim which directly activate Bax to trigger apoptosis. Mol Cell Biol 2002;22:3577-89.

32 Yamaguchi T, Okada T, Takeuchi K, et al. Enhancement of thymidine kinase-mediated killing of malignant glioma by BimS, a BH3-only cell death activator. Gene Ther 2003;10:375-85.

33 Brewis ND, Phelan A, Normand N, et al. Particle assembly incorporating a VP22-BH3 fusion protein, facilitating intracellular delivery, regulated release, and apoptosis. Mol Ther 2003;7:262-70

34 McNeish IA, Tenev T, Bell S, et al. Herpes simplex virus thymidine kinase/ganciclovir-induced cell death is enhanced by co-expression of caspase- 3 in ovarian carcinoma cells. Cancer Gene Ther 2001;8:308-19.

35 McNeish IA, Bell S, McKay T, et al. Expression of Smac/DIABLO in ovarian carcinoma cells induces apoptosis via a caspase-9-mediated pathway. Exp Cell Res 2003;286:186-98. 